\title{
Choosing Wisely: Experience of a Community Academic Hospital Pharmacy in Identifying Opportunities and Implementing Changes
}

\author{
Monica Lee, Jenny Chiu, and Edith Rolko
}

\section{INTRODUCTION}

$T^{1}$ The Choosing Wisely campaign was initiated in 2012 by the American Board of Internal Medicine Foundation, in partnership with Consumer Reports. The mandate was to engage patients and health care professionals in discussions about unnecessary tests and treatments, in an effort to make smart and effective care choices. National health care organizations representing medical specialties were asked to identify the "top 5 things to question" to improve quality that could be achieved through their individual professions. ${ }^{1}$ The basic principle of the campaign was to examine "waste's effect on quality of care, patient harm, and resource use." ${ }^{2}$ In the United States, the campaign began with 9 medical societies and has grown to more than 70 societies, with more than 400 evidence-based recommendations to date (late 2017). ${ }^{1}$ The Choosing Wisely Canada campaign was launched in April 2014 in partnership with the Canadian Medical Association and the University of Toronto, and by late 2017, over 250 recommendations had been developed by more than 40 societies. ${ }^{3}$ The objectives of Choosing Wisely Canada are outlined in Box $1 .^{4}$ This article

\section{Box 1. Objectives of the Choosing Wisely Canada Campaign $^{4}$ \\ 1. Encouraging physicians to engage in conversations with patients about overuse of tests, treatments, and medical procedures \\ 2. Empowering patients to make informed choices \\ 3. Cultivating a culture of responsible stewardship of health care resources \\ 4. Engendering public dialogue on the issue of "more is not always better" \\ 5. Engaging health system and non-medical stakeholders at all levels}

describes the preliminary experience of a pharmacy in a university-affiliated community hospital in implementing selected Choosing Wisely recommendations. The project was limited to the assessment, selection, and implementation of recommendations; evaluation of patient outcomes was beyond its scope.

\section{DESCRIPTION OF THE PROJECT}

\section{Choosing Wisely at North York General Hospital}

North York General Hospital (NYGH) is a 420-bed community academic hospital providing a wide range of acute care, ambulatory, and long-term care services. NYGH was one of the early adopters of Choosing Wisely Canada, launching its own campaign in June 2014. The hospital used 4 concurrent strategies to guide implementation of the campaign: leadership engagement, idea generation and evaluation, front-line staff engagement, and patient engagement (Figure 1).

Leadership engagement started with the development of a hospital-wide interprofessional Choosing Wisely Committee to support the campaign. The committee members consisted of representatives from the senior leadership team, medical chiefs, directors of clinical programs and departments, a patient advisor, and supporting departments such as Informatics and Corporate Communications. The committee provided clinical and administrative leadership by reviewing pertinent Choosing Wisely recommendations and identifying and prioritizing those with the most potential impact at NYGH, engaging staff, and leading hospital-wide initiatives. The first project involved examination of the utilization and appropriateness of laboratory tests and diagnostic imaging. Individual departments, including the Pharmacy Department, were asked to identify their lists of 


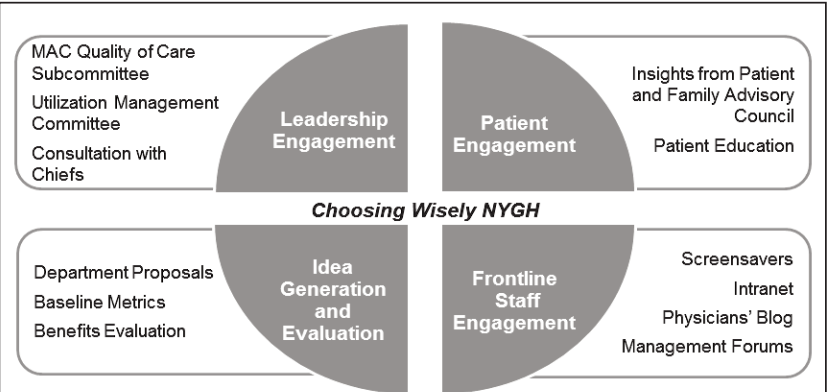

Figure 1. The 4 strategies used in implementation of the Choosing Wisely Canada Campaign at North York General Hospital. MAC = Medical Advisory Committee. Adapted with permission from North York General Hospital - ARTIC Choosing Wisely.

"top 5" recommendations. To encourage staff engagement, several hospital-wide initiatives were used, such as obtaining input from front-line staff for Choosing Wisely-type ideas; making reminder screensavers available; contributing to blogs; and posting reminders on the hospital intranet, in physician news bulletins, and social media. Patient and family engagement was an integral component of this Choosing Wisely campaign. The patient advisor on the NYGH Choosing Wisely Committee served as the liaison with the Patient and Family Advisory Council on the identified Choosing Wisely priorities, approaches to patient engagement, and communication of the Choosing Wisely campaign message.

\section{Medication-Related Choosing Wisely Recommendations}

In May 2014, the Pharmacy Department reviewed a total of 326 recommendations from 60 societies involved in the Choosing Wisely and Choosing Wisely Canada campaigns. Of the 52 medication-related recommendations, 28 were considered relevant to inpatients. On the basis of anecdotal observation of overutilization of certain classes of medications (from drug utilization data) and the perceived lack of benefit and/or safety of these medications, the pharmacy leadership team finalized the following 5 recommendations for implementation:

- Don't use stool softeners alone to prevent opioid-induced constipation. $^{5}$

- Don't maintain long-term proton pump inhibitor (PPI) therapy for gastrointestinal symptoms without an attempt to stop/reduce PPI at least once per year in most patients. ${ }^{6}$

- Don't take a multivitamin, vitamin E, or $\beta$-carotene to prevent cardiovascular disease or cancer. ${ }^{7}$

- Don't use benzodiazepines or other sedative-hypnotics in older adults as first choice for insomnia, agitation, or delirium. ${ }^{8}$
- Don't use antipsychotics as first choice to treat behavioural and psychological symptoms of dementia (BPSD). ${ }^{8}$

\section{Implementing the Choosing Wisely Recommendations}

Strategies for implementing Choosing Wisely recommendations consisted of formulary management and revision of electronic order sets. Proposed changes to the formulary underwent rigorous reviews by the Pharmacy and Therapeutics Committee and the Medical Advisory Committee. In addition, the hospital provided support to the Informatics Department in updating medication choices in electronic order sets and augmenting order sets with clinical notes based on Choosing Wisely recommendations.

The pharmacy leadership team (including the 3 authors of this report) led the medication-related Choosing Wisely initiatives. The organization equipped them with tools to carry out this project by enrolling them in knowledge translation educational programs. These educational events also gave the team leaders opportunities for networking with pharmacists from other institutions and sharing Choosing Wisely implementation experiences. Engagement of clinical pharmacists was achieved by providing educational sessions on the Choosing Wisely Campaign, the proposed medication-related initiatives, and their associated evidence-based resources. The clinical pharmacists were consulted to identify the potential impact of the initiatives and anticipated challenges with their implementation. The clinical pharmacists were also provided with resources such as patient information leaflets produced by Choosing Wisely Canada, which could be used when reviewing recommendations with patients.

Specific strategies for implementing each of the 5 Choosing Wisely recommendations are described below.

\section{Stool Softeners}

Although docusate products have been widely used for the management of constipation, a published review of the evidence showed that this drug is not more effective than placebo in the prevention or management of constipation. ${ }^{9}$ Guidelines on the management of constipation either omit stool softeners as options ${ }^{10}$ or describe low evidence for their use. ${ }^{11}$ At NYGH, docusate products were on the formulary and were available in 55 order sets. After completion of an evidencebased class review of laxatives at NYGH, the pharmacy leadership group recommended that docusate be removed from the order sets to prevent initiation of this medication. This suggestion was approved by the Medical Advisory Committee in April 2015 and implemented in September 2015. Order sets for constipation management were updated to guide prescribing of more effective alternatives, such as osmotic or stimulant 
laxatives. Use of docusate has declined steadily over time (Figure 2 ), and the drug was eventually removed from the formulary in February 2017. The effect of this change in recommendation on patient outcomes has not yet been evaluated.

\section{Proton Pump Inhibitors}

Several guidelines, ${ }^{12,13}$ including those of Choosing Wisely Canada,${ }^{6}$ have recommended against long-term PPI therapy for gastrointestinal symptoms without attempts to reduce or stop therapy, except for high-risk patients. Risks that have been reported to be associated with PPI therapy include communityacquired pneumonia, fractures, Clostridium difficile infections, vitamin $\mathrm{B}_{12}$ deficiency, and hypomagnesemia. ${ }^{13}$ A 5-month deprescribing pilot was conducted at NYGH between March and August 2016 on a medicine unit where patients have been deemed medically stable and designated as needing an alternate level of care (ALC). ALC patients were selected for this pilot because attempted deprescribing of PPI was considered more appropriate for patients in whom all acute medical issues had been resolved. The unit pharmacist evaluated the indication for PPI in each patient and, using the PPI deprescribing algorithm as a guide, ${ }^{13}$ determined whether deprescribing should be proposed. The pharmacist then discussed the deprescribing recommendations with the patient, using Choosing Wisely patient information leaflets or similar resources. The interdisciplinary team monitored the patient during the deprescribing process until the time of discharge. The deprescribing intervention and follow-up plan were conveyed to the next health care provider through the discharge information package. Pharmacists made a deprescribing recommendation for 20 patients; of these, $12(60 \%)$ were accepted, resulting in PPI discontinuation for 10 patients and dose reductions for 2 patients. ${ }^{14}$ Although attempting PPI deprescribing could be a

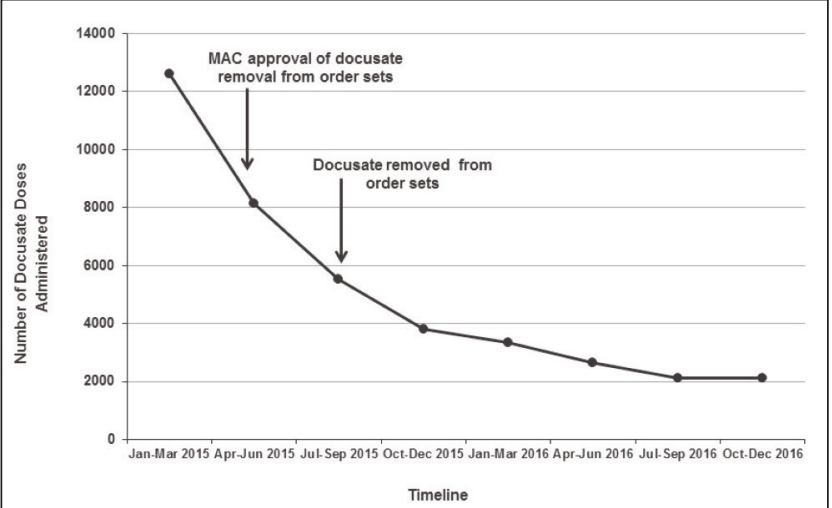

Figure 2. Use of docusate before and after removal from all 55 hospital order sets that originally included this drug. Each data point represents a 3-month period (one quarter). MAC = Medical Advisory Committee. challenge in an acute care setting, deprescribing of these drugs can be successfully implemented in selected patients (e.g, those designated as ALC). These strategies can be used for ALC patients in other units of the hospital or in similar populations in other hospitals.

\section{Multivitamins}

Vitamin and mineral supplements are commonly used. One study reported that $40.1 \%$ of adult Canadians took supplements. ${ }^{15}$ Although discontinuation of multivitamins is not a current Choosing Wisely Canada recommendation, the American College of Preventive Medicine has suggested as 1 of its top 5 recommendations, "Don't take a multi-vitamin, vitamin $\mathrm{E}$ or beta-carotene to prevent cardiovascular disease or cancer." The pharmacy leadership group at NYGH discussed the role of vitamins and minerals in the hospital setting as an idea for the Choosing Wisely campaign. The concern was the widespread use of multivitamin products and a lack of evidence for their use. ${ }^{16-18}$ Additionally, multivitamins that contain minerals pose a risk of drug interactions, thus generating the need for rescheduling medication administration times. An audit showed that some patients were started on multivitamin therapy through order sets. This observation prompted a review of all order sets containing an order for oral multivitamin. It was found that 19 order sets had a default for oral multivitamin. The CPOE [Computerized Provider Order Entry] Order Set Team worked with the clinical dietitians to assess the need for default multivitamin orders, and eventually had the default setting removed from 10 of the order sets. Examples of order sets from which the multivitamin default was removed were admission order sets in general medical care and for patients with stroke, seizures, or abdominal pain. The default was retained in order sets where multivitamin therapy was considered appropriate, such as those for patients admitted because of alcohol withdrawal, patients known to have significant alcohol use, and patients with eating disorders or requiring enteral nutritional support. Use of multivitamins has decreased by about 19\% since this change (Figure 3).

\section{Benzodiazepines}

The adverse effects associated with benzodiazepines are well documented. These drugs are associated with an increased risk of falls, hip fractures, motor vehicle accidents, and cognitive impairment. ${ }^{8}$ Prescribing or discontinuing these medications in hospital may have a substantial impact on their long-term use. ${ }^{8}$ Choosing Wisely Canada recommends that benzodiazepines and other sedative-hypnotics not be used in older adults as the first choice for insomnia, agitation, or delirium. ${ }^{8}$

There is no therapy for the treatment of insomnia that is both safe and effective. To provide a safer alternative to 


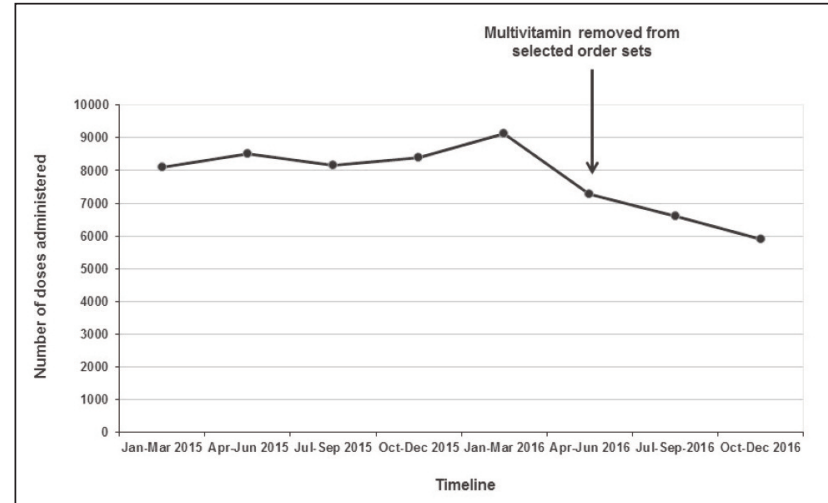

Figure 3. Use of multivitamin tablets before and after removal from 10 of the 19 hospital order sets that originally included this drug. Each data point represents a 3-month period (one quarter).

benzodiazepines, melatonin was added to the NYGH drug formulary in April 2015. Although the evidence regarding its efficacy is not robust, melatonin was at least partially effective in half of the elderly inpatients included in an internal retrospective chart review evaluation. ${ }^{19}$ Insomnia order sets were modified to remove all benzodiazepine options, which prevented patients from being started and discharged on this class of medications. Melatonin and nonpharmacological measures such as ear plugs were added to the order sets. Additional warnings were included in the order sets to remind clinicians that benzodiazepines should not be used in the management of delirium, except in cases of alcohol or benzodiazepine withdrawal. Further analyses will be undertaken at NYGH to evaluate the trend in use of benzodiazepines, other hypnotics, and melatonin in the management of insomnia in hospitalized older adults. In particular, the number of orders by patient-days for each class of medications will be compared before and after the order set changes.

\section{Antipsychotics}

Antipsychotics are associated with increased risks of mortality and cardiovascular events when used in individuals with dementia. ${ }^{20,21}$ The benefit of antipsychotics in the management of BPSD $^{22,23}$ and delirium ${ }^{24}$ is limited. As such, the use of antipsychotics should be reserved for cases in which nonpharmacological measures have failed and patients pose an imminent threat to themselves or others. ${ }^{8}, 12$ Deprescribing of antipsychotics has been a goal for ALC patients at NYGH. The unit pharmacist worked with the physicians and the interprofessional team to identify patients for whom antipsychotics had been prescribed for BPSD or delirium. If the use of antipsychotics was deemed necessary, the antipsychotic therapy was reassessed on a regular basis for tapering and discontinuation whenever it was possible. At NYGH, nonpharmacological approaches, such as the Montessori Method, gentle persuasion, and music therapy have been preferred over pharmacotherapy; the hospital has found these nonpharmacological approaches to be effective in individual cases after antipsychotics have been successfully tapered off. Although formal research studies have not been conducted, preliminary observations by the unit pharmacist have indicated that deprescribing of antipsychotics was successful in about $80 \%$ of the patients with initiation of treatment for delirium, because the condition eventually resolved and antipsychotics were no longer required. Among patients with BPSD, however, the success rate for tapering off antipsychotics has been closer to $60 \%$.

\section{IMPLICATIONS AND SIGNIFICANCE FOR PRACTICE}

The successful adoption of Choosing Wisely initiatives at NYGH has largely been attributed to organization-wide support, stakeholder education, and availability of resources. The NYGH Choosing Wisely Committee is dedicated to providing ongoing clinical and leadership support for this campaign, by meeting regularly to develop quality improvement projects to help sustain new initiatives. Communication of key milestones, identification of benefits achieved, and implementation of new initiatives are key elements for continuing staff engagement. Furthermore, collaboration with other institutions and agencies (e.g., government) may help to accelerate the implementation and spread of proven research evidence into broader practice. Since April 2016, NYGH has been the lead site, in partnership with the Adopting Research to Improve Care (ARTIC) program, in a project to disseminate NYGH Choosing Wisely knowledge and experience to 4 member hospitals and 6 affiliated family health teams. ${ }^{25}$

The process of reviewing Choosing Wisely recommendations, drug utilization data, and practices within the hospital helped the Pharmacy Department to focus on minimizing the use of a selected group of unnecessary drug therapies. Several challenges were recognized during the preliminary implementation process. Not all Choosing Wisely recommendations are appropriate for adoption in the acute care setting. Therefore, successful adoption of Choosing Wisely Canada recommendations will require a proper feasibility assessment and use of multimodal strategies. Furthermore, certain Choosing Wisely recommendations are suitable for centre-wide implementation, whereas others are appropriate only for selected groups of patients. Our experience in adopting Choosing Wisely recommendations showed that the effects of each recommendation may vary, depending on factors such as cultural shifts and stakeholders' buy-in. Pharmacists may need more time for some interventions, such as PPI deprescribing, if the interventions involve identifying indications for particular medications to determine the appropriateness of deprescribing in individual 


\section{Box 2. Choosing Wisely Recommendations from Various Pharmacy Societies}

\section{Society of Hospital Pharmacists of Australia*}

1. Don't initiate and continue medicines for primary prevention in individuals who have a limited life expectancy.

2. Don't initiate an antibiotic without an identified indication and a predetermined length of treatment or review date.

3. Don't initiate and continue antipsychotic medicines for behavioural and psychological symptoms of dementia for more than 3 months.

4. Don't recommend the regular use of oral nonsteroidal anti-inflammatory medicines (NSAIDs) in older people.

5. Don't recommend the use of medicines with subtherapeutic doses of codeine ( $<30 \mathrm{mg}$ for adults) for mild to moderate pain.

\section{American Society of Health-System Pharmacistst}

1. Do not initiate medications to treat symptoms, adverse events, or side effects without determining if an existing therapy or lack of adherence is the cause, and whether a dosage reduction, discontinuation of a medication, or another medication is warranted.

2. Do not prescribe medications for patients on five or more medications, or continue medications indefinitely, without a comprehensive review of their existing medications, including over-the-counter medications and dietary supplements, to determine whether any of the medications or supplements should or can be discontinued.

3. Do not continue medications based solely on the medication history unless the history has been verified with the patient by a medication-use expert (e.g., a pharmacist) and the need for continued therapy has been established.

4. Do not prescribe patients medications at discharge that they were on prior to admission without verifying that these medications are still needed and that the discharge medications will not result in duplication, drug interactions, or adverse events.

5. Do not prescribe or administer oral liquid medications using teaspoon or tablespoon for measurement; use only milliliters ( $\mathrm{mL}$ ) when measuring with an approved dosing device (e.g., medication cup or oral syringe).

*Reproduced, with permission, from the Society of Hospital Pharmacists of Australia. ${ }^{27}$

tReproduced, with permission, from "Five Things Physicians and Patients Should Question"28

www.choosingwisely.org/societies/american-society-of-health-system-pharmacists (June 1, 2017). @ 2017, American Society of Health-System Pharmacists, Inc. All rights reserved.

patients. Although it is important for an organization to have ongoing evaluation of the effects of changes implemented, meaningful evaluation of patient outcomes requires additional staff resources and time, which may not always be available. Patient outcomes and satisfaction with care were not evaluated in the current study.

\section{PHARMACISTS' ROLE AND FUTURE DIRECTION}

Pharmacy societies in Canada have yet to identify the "top 5" Choosing Wisely recommendations, although the Canadian Pharmacists Association has asked pharmacists for their input on development of such a list. ${ }^{26}$ To date, 2 pharmacy societies, the Society of Hospital Pharmacists of Australiaa ${ }^{27}$ and the American Society of Health-System Pharmacists, ${ }^{28}$ have contributed official Choosing Wisely recommendations. These 2 sets of recommendations are presented in Box 2.

Pharmacists are well positioned to promote the Choosing Wisely campaign. As providers of pharmaceutical care, we ensure, to the extent feasible, that all patients' medications are indicated, effective, safe, and convenient to take. Our endeavours in medication review and medication reconciliation, stewardship programs, and deprescribing initiatives can be used as starting points in identifying practices that we should question. As such, we may want to adopt the motto "Don't fill it before you talk about it."

\section{References}

1. Choosing Wisely: about. Philadelphia (PA): Choosing Wisely; 2017 [cited 2017 May 10]. Available from: www.choosingwisely.org/about-us/

2. Wolfson D, Santa J, Slass L. Engaging physicians and consumers in conversations about treatment overuse and waste: a short history of the Choosing Wisely campaign. Acad Med. 2014;89(7):990-5.

3. About Choosing Wisely Canada. Toronto (ON): Choosing Wisely Canada; 2017 [cited 2017 Sep 2]. Available from: https:/choosingwiselycanada.org/ about/

4. Communication toolkit. Toronto (ON): Choosing Wisely Canada; 2014 [cited 2017 Jan 6]. Available from: https://rheum.ca/images/documents/Choosing Wisely_Communications_Toolkit_FINAL.pdf

5. Palliative care: five things physicians and patients should question. Toronto $(\mathrm{ON})$ : Choosing Wisely Canada; [updated 2014 Oct 29; cited 2017 Jan 15]. Available from: www.choosingwiselycanada.org/recommendations/palliative-care/

6. Gastroenterology: five things physicians and patients should question. Toronto (ON): Choosing Wisely Canada; [updated 2014 Oct 29; cited 2017 Jan 15]. Available from: www.choosingwiselycanada.org/recommendations/gastroenterology-2/

7. American College of Preventive Medicine: five things physicians and patients should question. Philadelphia (PA): Choosing Wisely; 2015 [cited 2017 Jan 15]. Available from: www.choosingwisely.org/societies/american-college-ofpreventive-medicine/

8. Geriatrics: five things physicians and patients should question. Toronto $(\mathrm{ON})$ : Choosing Wisely Canada; [updated 2014 Apr 2; cited 2017 Jan 15]. Available from: www.choosingwiselycanada.org/recommendations/geriatrics/

9. Dioctyl sulfosuccinate or docusate (calcium or sodium) for the prevention or management of constipation: a review of the clinical effectiveness [rapid response report: summary with critical appraisal]. Ottawa $(\mathrm{ON})$ : Canadian Agency for Drugs and Technologies in Health; 2014 [cited 2016 Dec 8]. Available from: https:/www.cadth.ca/sites/default/files/pdf/htis/nov-2014/ RC0561\%20Stool\%20Softeners\%20Final.pdf

10. American Gastroenterological Association; Bharucha AE, Dorn SD, Lembo A, Pressman A. American Gastroenterological Association medical position statement on constipation. Gastroenterology. 2013;144(1):211-7. 
11. Paré P. The approach to diagnosis and treatment of chronic constipation: suggestions for a general practitioner. Can J Gastroenterol. 2011;25 Suppl B:36B-40B.

12. American Geriatrics Society 2015 Beers Criteria Update Expert Panel. American Geriatrics Society 2015 updated Beers criteria for potentially inappropriate medication use in older adults. J Am Geriatr Soc. 2015;63(11): 2227-46.

13. Evidence-based deprescribing algorithm for proton pump inhibitors. Kitchener (ON): Ontario Pharmacy Evidence Network; 2015 [cited 2017 Jan 21]. Available from: www.open-pharmacy-research.ca/evidence-based-ppideprescribing-algorithm

14. Wang S, Lee M. Applying the proton pump inhibitor deprescribing algorithm in stable patients in a community hospital [abstract]. Can J Hosp Pharm. 2017; $70(1): 86$.

15. Guo X, Willows N, Kuhle S, Jhangri G, Veugelers PJ. Use of vitamin and mineral supplements among Canadian adults. Can J Public Health. 2009; 100(5):357-60.

16. Fortmann SP, Burda BU, Senger CA, Lin JS, Whitlock EP. Vitamin and mineral supplements in the primary prevention of cardiovascular disease and cancer: an updated systematic evidence review for the U.S. Preventive Services Task Force. Ann Intern Med. 2013;159(12):824-34.

17. Grodstein F, O'Brien J, Kang JH, Dushkes R, Cook NR, Okereke O, et al. Long-term multivitamin supplementation and cognitive function in men: a randomized trial. Ann Intern Med. 2013;159(12):806-14.

18. Lamas GA, Boineau R, Goertz C, Mark DB, Rosenberg Y, Stylianou M, et al; TACT (Trial to Assess Chelation Therapy) Investigators. Oral high-dose multivitamins and minerals after myocardial infarction: a randomized trial. Ann Intern Med. 2013;159(12):797-805.

19. Lee M, Ghazi L. Evaluation of melatonin use for the management of insomnia in elderly inpatients in a community hospital [abstract]. Can J Hosp Pharm. 2017;70(1):66.

20. Schneider LS, Dagerman KS, Insel P. Risk of death with atypical antipsychotic drug treatment for dementia: meta-analysis of randomized placebo-controlled trials. JAMA. 2005;294(15):1934-43.

21. Trifirò G, Spina E, Gambassi G. Use of antipsychotics in elderly patients with dementia: do atypical and conventional agents have a similar safety profile? Pharmacol Res. 2009;59(1):1-12.

22. Maher AR, Maglione M, Bagley S, Suttorp M, Hu JH, Ewing B, et al. Efficacy and comparative effectiveness of atypical antipsychotic medications for off-label uses in adults: a systematic review and meta-analysis. JAMA. 2011;306(12):1359-69.

23. Schneider LS, Tariot PN, Dagerman KS, Davis SM, Hsiao JK, Ismail MS, et al.; CATIE-AD Study Group. Effectiveness of atypical antipsychotic drugs in patients with Alzheimer's disease. N Engl J Med. 2006;355(15): 1525-38.

24. Neufeld KJ, Yue J, Robinson TN, Inouye SK, Needham DM. Antipsychotic medication for prevention and treatment of delirium in hospitalized adults: a systematic review and meta-analysis. J Am Geriatr Soc. 2016; 64(4):705-714.
25. ARTIC program announces newest project: Choosing Wisely - an idea worth spreading. Toronto (ON): Health Quality Ontario; 2016 [cited 2017 Jan 15]. Available from: www.hqontario.ca/portals/0/documents/qi/artic/news-releasechoosing-wisely-160407-en.pdf

26. $\mathrm{CPhA}$ calls for input from pharmacist community to create Choosing Wisely Canada recommendations. Ottawa $(\mathrm{ON})$ : Canadian Pharmacists Association; 2016 Nov 24 [cited 2017 Sep 7]. Available from: https://www.pharmacists.ca/ news-events/news/cpha-calls-for-input-from-pharmacist-community-tocreate-choosing-wisely-canada-recommendations/

27. SHPA's list of Choosing Wisely Australia recommendations. Collingwood (Australia): Society of Hospital Pharmacists of Australia; 2016 [cited 2017 Jan 6]. Available from: https://www.shpa.org.au/shpas-list-of-choosing-wiselyaustralia-recommendations

28. American Society of Health-System Pharmacists: five things physicians and patients should question. Philadelphia (PA): Choosing Wisely; 2017 Jun 1 [cited 2017 Sep 5]. Available from: www.choosingwisely.org/societies/americansociety-of-health-system-pharmacists/

Monica Lee, BScPhm, MSc, PharmD, RPh, is Elder Care Pharmacy Practitioner with North York General Hospital and an Adjunct Lecturer with the Leslie Dan Faculty of Pharmacy, University of Toronto, Toronto, Ontario.

Jenny Chiu, BSCPhm, PharmD, ACPR, RPh, is an Acute Care Practitioner/Clinical Coordinator with North York General Hospital and an Adjunct Lecturer with the Leslie Dan Faculty of Pharmacy, University of Toronto, Toronto, Ontario.

Edith Rolko, BScPhm, RPh, is Director of Pharmacy with North York General Hospital and an Adjunct Professor with the Leslie Dan Faculty of Pharmacy, University of Toronto, Toronto, Ontario.

Competing interests: None declared.

\section{Address correspondence to:}

Dr Monica Lee

North York General Hospital

4001 Leslie Street

Toronto ON M2K $1 \mathrm{E} 1$

e-mail: monica.lee@nygh.on.ca

Funding: None received.

Acknowledgement: The authors are grateful for presubmission review of the manuscript by Marwan Asalya, Project Manager, ARTIC Choosing Wisely, North York General Hospital. 